\title{
Health Assessment Questionnaire-Disability Index (HAQ-DI) use in modelling disease progression in diffuse cutaneous systemic sclerosis: an analysis from the EUSTAR database
}

Yannick Allanore ${ }^{1 *}$ (D) Sylvie Bozzi ${ }^{2}$, Augustin Terlinden², Doerte Huscher ${ }^{3}$, Caroline Amand ${ }^{2}$, Christina Soubrane ${ }^{2}$, Elise Siegert ${ }^{4}$, László Czirják ${ }^{5}$, Patricia E. Carreira ${ }^{6}$, Eric Hachulla ${ }^{7}$, Elisabetta Zanatta ${ }^{8}$, Mengtao Li ${ }^{9}$, Paolo Airò ${ }^{10}$, Fabian A. Mendoza ${ }^{11}$, Edoardo Rosato ${ }^{12}$, Oliver Distler ${ }^{13}$ and on behalf of the EUSTAR Collaborators

\footnotetext{
Abstract

Background: Patients with diffuse cutaneous systemic sclerosis (dcSSc) have a poor prognosis. The importance of monitoring subjective measures of functioning and disability, such as the Health Assessment QuestionnaireDisability Index (HAQ-DI), is important as dCSSC is rated by patients as worse than diabetes or hemodialysis for quality of life impairment. This European Scleroderma Trials and Research (EUSTAR) database analysis was undertaken to examine the importance of impaired functionality in dcSSc prognosis. The primary objectives were to identify predictors of death and HAQ-DI score progression over 1 year. HAQ-DI score, major advanced organ involvement, and death rate were also used to develop a comprehensive model to predict lifetime dcSSc progression.

Methods: This was an observational, longitudinal study in patients with dcSSc registered in EUSTAR. Death and HAQ-DI scores were, respectively, analyzed by Cox regression and linear regression analyses in relation to baseline covariates. A microsimulation Markov model was developed to estimate/predict natural progression of dcSSc over a patient's lifetime.

(Continued on next page)
}

\footnotetext{
* Correspondence: yannick.allanore@aphp.fr

${ }^{1}$ Service de Rhumatologie, Cochin Hospital, APHP, Paris Descartes University, 27 rue du Faubourg Saint Jacques, 75014 Paris, France

Full list of author information is available at the end of the article
}

(c) The Author(s). 2020 Open Access This article is licensed under a Creative Commons Attribution 4.0 International License, which permits use, sharing, adaptation, distribution and reproduction in any medium or format, as long as you give appropriate credit to the original author(s) and the source, provide a link to the Creative Commons licence, and indicate if changes were made. The images or other third party material in this article are included in the article's Creative Commons licence, unless indicated otherwise in a credit line to the material. If material is not included in the article's Creative Commons licence and your intended use is not permitted by statutory regulation or exceeds the permitted use, you will need to obtain permission directly from the copyright holder. To view a copy of this licence, visit http://creativecommons.org/licenses/by/4.0/ The Creative Commons Public Domain Dedication waiver (http://creativecommons.org/publicdomain/zero/1.0/) applies to the data made available in this article, unless otherwise stated in a credit line to the data. 
(Continued from previous page)

Results: The analysis included dcSSc patients with $(N=690)$ and without $(N=4132) \mathrm{HAQ-DI}$ score assessments from the EUSTAR database. Baseline HAQ-DI score, corticosteroid treatment, and major advanced organ involvement were predictive of death on multivariable analysis; a 1-point increase in baseline HAQ-DI score multiplied the risk of death by $2.7(p<0.001)$ and multiple advanced major organ involvement multiplied the risk of death by 2.8 ( $p<0.05$ ). Multivariable analysis showed that baseline modified Rodnan Skin Score (mRSS) and baseline HAQ-DI score were associated with HAQ-DI score progression at 1 year $(p<0.05)$, but there was no association between baseline organ involvement and HAQ-DI score progression at 1 year. HAQ-DI score, major advanced organ involvement, and death were successfully used to model long-term disease progression in dcSSc.

Conclusions: HAQ-DI score and major advanced organ involvement were comparable predictors of mortality risk in dcSSc. Baseline mRSS and baseline HAQ-DI score were predictive of HAQ-DI score progression at 1 year, indicating a correlation between these endpoints in monitoring disease progression. It is hoped that this EUSTAR analysis may change physician perception about the importance of the HAQ-DI score in dcSSc.

Keywords: EUSTAR registry, Diffuse cutaneous systemic sclerosis, Health Assessment Questionnaire-Disability Index, HAQ-DI score

\section{Introduction}

Systemic sclerosis (SSc) is an autoimmune disorder characterized by extensive fibrosis and vasculopathy that can affect the skin and internal organs [1]. It imposes a substantial burden of pain, disfigurement, and impaired functionality that can markedly reduce health-related quality of life [2, 3]. SSc is a heterogeneous disease, and it is commonly subclassified according to the extent of skin involvement [4]. Patients with diffuse cutaneous systemic sclerosis $(\mathrm{dcSSc})$ have the poorest prognosis, with a 10-year mortality rate of at least 50\% [5]. It is, therefore, important to monitor these patients in order to provide a comprehensive and long-term assessment of disease progression.

To date, the modified Rodnan Skin Score (mRSS) has been widely used to monitor progression in clinical studies; it is a semi-quantitative measure of skin thickness in different body areas that is used as a proxy for disease severity, progression, and mortality risk [6, 7]. Shortterm progressive skin fibrosis (i.e., within 1 year measured via mRSS) is associated with a later decline in lung function and worse survival in patients with dcSSc [7]. However, the progression of skin fibrosis diverges from other organ involvement during the disease course. It often regresses spontaneously once it has reached its peak, while lung fibrosis continues to progress in most cases. This can make short-term clinical trials of therapeutic agents challenging as outcomes reported with the mRSS do not always correlate with other objective measures $[8,9]$.

Ensuring that the patient voice is heard is also central to decision making. Patients with SSc have strong views about the chronic nature and negative consequences of their disease. They perceive the impact of their disease on daily living as more severe than patients with diabetes or those undergoing hemodialysis [10]. However, patient opinion is often not satisfactorily included in decision making [2]. Patient-reported outcomes have become increasingly important in recent years; they could address this imbalance and increase our understanding of the wider impact of these diseases.

Accumulating evidence suggests that the Health Assessment Questionnaire-Disability Index (HAQ-DI) is a useful measure of functionality in SSc [11-13]. This is a self-reported questionnaire covering 20 items in eight domains related to measuring difficulty in performing activities of daily living: dressing, arising, eating, walking, hygiene, reach, grip, and common daily activities [12, 13]. Each question is rated on a $0-3$ scale, where 0 indicates "without difficulty" and 3 indicates "unable to do," and additional points can be added if aids or devices are needed for specific activities; thus, increasing score indicates worse functionality $[12,13]$. HAQ-DI score is a reliable measure that is sensitive to change in disease activity in cross-sectional (patients and physicians) and longitudinal studies $[2,12]$. As a result, it is now increasingly used as an endpoint in clinical studies investigating treatment outcomes in SSc.

The primary aims of this European Scleroderma Trials and Research (EUSTAR) database analysis of patients with dcSSc were (1) to identify predictors of death including HAQ-DI score, advanced major organ involvement, and other clinical characteristics; (2) to identify predictors of HAQ-DI score progression over 1 year; and (3) to develop a transition model to predict natural progression of dcSSc over a lifetime using HAQ-DI score, major advanced organ involvement, and death rates.

\section{Methods}

\section{Design and study population}

This was an observational, longitudinal study of patients with dcSSc registered in the EUSTAR database. The 
EUSTAR network has been described elsewhere [14, 15]. In brief, EUSTAR is a growing database of patients with scleroderma treated at centers worldwide; all patients undergo annual scheduled clinic visits, providing observational, longitudinal data.

For this analysis, patient data were extracted from January 1995 to February 2019. Ethics Committee approval was obtained from all centers, and informed consent was provided when required by the ethical regulations at the specific centers. Patients were $\geq 18$ years of age and to have SSc as classified by the American College of Rheumatology (ACR) (1980) or ACR/ European League Against Rheumatism criteria (2013) $[16,17]$. Patients with dcSSc were identified from this cohort based on the LeRoy criteria [4], and those with the available data for the first non-Raynaud's manifestation were extracted. If data on the LeRoy criteria were not available, then the extent of skin involvement (e.g., skin fibrosis at any time with $\mathrm{mRSS} \geq 1$ of upper arms, thorax, abdomen, or thighs) was used as a surrogate.

\section{Population and outcomes}

Analyses were performed using patients with dcSSc who met the above criteria with or without HAQ-DI score assessments. Patients with HAQ-DI score were further divided into either those who had $\geq 1 \mathrm{HAQ}$-DI or those who had $\geq 2$ HAQ-DI score assessments. Clinical data used in analyses are shown in Table 1, Fig. 1, and Fig. S1.

Analyses of the impact of organ involvement focused on the major advanced complications seen in dcSSc patients. Advanced gastrointestinal (GI) events were

Table 1 Baseline demographic and clinical characteristics according to HAQ-DI score assessments

\begin{tabular}{|c|c|c|c|}
\hline Parameters & $\begin{array}{l}\text { dcSSc with no HAQ-DI score } \\
\text { at any time } \\
N=4132\end{array}$ & $\begin{array}{l}\text { dcSSc with } \geq 1 \text { HAQ-DI } \\
\text { score } \\
N=690\end{array}$ & $\begin{array}{l}\text { dcSSc with } \geq 2 \text { HAQ-DI } \\
\text { scores } \\
N=424\end{array}$ \\
\hline \multicolumn{4}{|l|}{ Age (years) } \\
\hline Mean (SD) & $52.2(13.6)$ & $53.8(12.9)$ & $53.1(12.7)$ \\
\hline IQR & $42.6-62.4$ & $45.6-62.6$ & $43.9-62.3$ \\
\hline Age (years) at onset of RP, $N$ & 3953 & 677 & 414 \\
\hline Mean (SD) & $43.3(14.7)$ & $42.4(14.4)$ & $42.5(14.3)$ \\
\hline IQR & $32.7-54.0$ & $32.4-52.0$ & $32.1-52.3$ \\
\hline Female, $n(\%)$ & $3214(77.8 \%)$ & $537(77.8 \%)$ & $320(75.5 \%)$ \\
\hline \multicolumn{4}{|l|}{ Disease duration, $n(\%)$} \\
\hline$\leq 18$ months & $962(23.3 \%)$ & $60(8.7 \%)$ & $39(9.2 \%)$ \\
\hline$>18$ months & 3170 (76.7\%) & $630(91.3 \%)$ & $385(90.8 \%)$ \\
\hline \multicolumn{4}{|l|}{ Current digital ulcers, $n(\%)$} \\
\hline Yes & 1493 (36.6\%) & $120(18.3 \%)$ & $70(17.3 \%)$ \\
\hline No & $2585(63.4 \%)$ & $535(81.7 \%)$ & $335(82.7 \%)$ \\
\hline \multicolumn{4}{|l|}{ Puffy fingers, $n(\%)$} \\
\hline Yes & $972(48.7 \%)$ & $216(35.5 \%)$ & $148(39.1 \%)$ \\
\hline No & 1024 (51.3\%) & $392(64.5 \%)$ & $231(60.9 \%)$ \\
\hline mRSS, N & 3895 & 656 & 403 \\
\hline Mean (SD) & $16.4(9.9)$ & $11.7(9.0)$ & $11.8(8.6)$ \\
\hline IQR & $9.0-23.0$ & $5.0-17.0$ & $5.0-17.0$ \\
\hline \multicolumn{4}{|l|}{ HAQ-DI score } \\
\hline Mean (SD) & NA & $1.0(0.8)$ & $1.1(0.8)$ \\
\hline IQR & NA & $0.4-1.6$ & $0.4-1.8$ \\
\hline \multicolumn{4}{|c|}{ Advanced organ involvement, $n$ (\%) } \\
\hline None & 3448 (83.7\%) & $468(68.8 \%)$ & $295(69.9 \%)$ \\
\hline One & $585(14.2 \%)$ & $178(26.2 \%)$ & $108(25.6 \%)$ \\
\hline Multiple & $86(2.1 \%)$ & $34(5.0 \%)$ & $19(4.5 \%)$ \\
\hline Missing & 13 & 10 & 2 \\
\hline
\end{tabular}




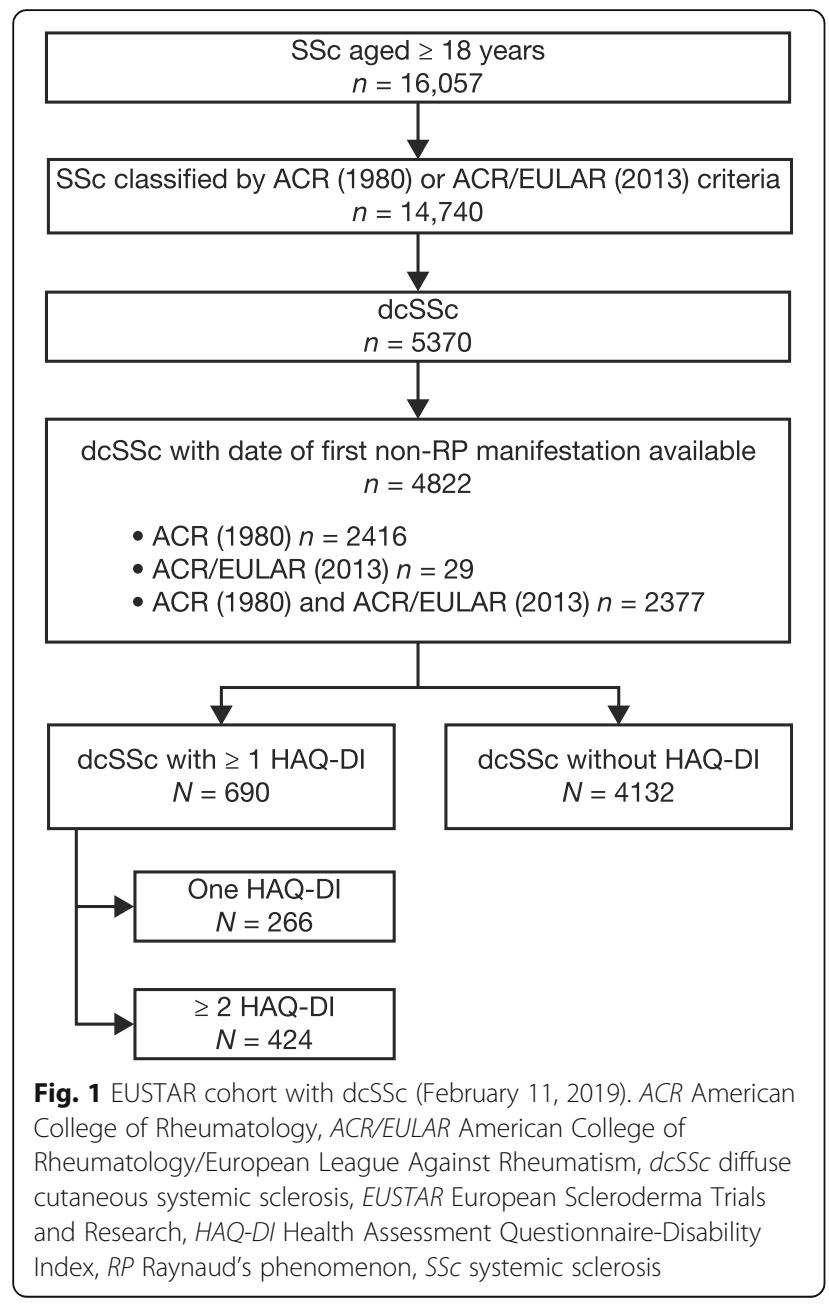

defined as malabsorption or $\geq 10 \%$ weight loss from baseline. Echocardiographic measurement of systolic pulmonary arterial pressure $>45 \mathrm{mmHg}$ was used as a proxy for pulmonary hypertension $(\mathrm{PH})$ as data on right heart catheterization were not collected routinely in the database. Interstitial lung disease (ILD) was confirmed by high-resolution computed tomography or chest Xray, and significant lung involvement was defined as ILD on imaging and forced vital capacity $<75 \%$ predicted. Cardiac involvement was defined as left ventricular ejection fraction (LVEF) $\leq 45 \%$ measured on echocardiography, and renal involvement was defined as the presence of renal crisis (abrupt onset of severe hypertension accompanied by rapidly progressive renal failure, hypertensive encephalopathy, congestive heart failure, and/or microangiopathic hemolytic anemia).

Patients could be enrolled at any time in the disease course if they were classified as having SSc (defined as baseline). Clinical history, demographic characteristics, use of immunomodulatory treatment, and death were documented for patients with or without HAQ-DI score assessments; this information was based on medical records in the EUSTAR database. It was intended that patients should be documented once a year. Death was documented with the date or year (if the date was not exactly known) and reason of death.

\section{Statistical analyses \\ Regression analyses to identify predictors of death and $H A Q-D I$ score progression}

Patients with $\geq 2$ HAQ-DI score assessments were selected to model prediction of HAQ-DI score at 1 year (i.e., progression). Patients with $\geq 2$ specific organ assessments were selected to predict major advanced organ involvement. Using these populations, the relationship between baseline characteristics and death rates and HAQ-DI score progression at 1 year were examined both at the univariate level and in multivariable models. Death rates and HAQDI scores were analyzed by Cox regression and linear regression analyses, respectively, in relation to each baseline covariate: gender, age at onset of Raynaud's phenomenon, RNA polymerase III positive, immunomodulator treatment, corticosteroid use $>10 \mathrm{mg} /$ day, HAQ-DI score, mRSS, and number of major advanced organs involved $(0,1$, or $\geq 2$ based on lung, cardiac, GI, PH, or renal involvement). All analyses were performed using $\mathrm{IBM}^{\circ}$ SPSS statistics 24.0 and R software (version 3.4.4).

\section{Transition model for dcSSc}

A microsimulation Markov model was developed to determine the natural progression of dcSSc over a patient's lifetime horizon. The Markov model was chosen as it enables simulation of a patient in different health states (e.g., various HAQ-DI levels, organ involvement or not, and alive or dead). Each patient was modelled independently to allow for the heterogeneity of the dcSSc population; it was, therefore, a microsimulation. Within the abovementioned dataset, three risk equations were developed to model the evolution of the following endpoints: HAQ-DI score; major advanced organ involvement; death.

The HAQ-DI score is a continuous variable and was transformed into a categoric variable in this model to represent five different health states ([0-0.5], [0.5-1.0], $[1.0-1.5],[1.5-2.0]$, and $[2.0-3.0])$; the health states were based on expert opinion and were initially defined as uniformly splitting the $0-3$ HAQ-DI score range into six categories (i.e., this is the maximum amount of categories that enable the multistate modelling [MSM] package to converge when fitting a transition matrix between HAQ-DI states); however, the two last categories did not contain enough patients and were grouped into a single state, leaving five states in total. The HAQ-DI transition matrix was calibrated using an MSM that provides constant transition probabilities over time from any HAQ-DI state to any other one. For calibration of 
the HAQ-DI transition matrix, transition intensities were developed using longitudinal, patient-level data for HAQ-DI states as a function of gender, age at baseline, and lung status at baseline. A 1-year cycle was chosen to transform intensities into yearly transition probabilities of moving between the five HAQ-DI states (Table S1).

For calibration of the organ equations, longitudinal patient-level data were used to calculate survival in the no-organ-involved state in relation to specific patient characteristics at baseline (age, sex, HAQ-DI states, and major advanced organ involvement such as lung, $\mathrm{PH}$, cardiac, renal, or GI) (Table S2). This equation was calibrated using the survival package ( $\mathrm{R}$ software).

For calibration of the third equation, death rates were calculated using a standardized mortality ratio (SMR), defined as the ratio of observed deaths in the dcSSc EUSTAR population to expected deaths in the general population (adjusted for age- and gender-specific rates) (Fig. 2). Thus, SMRs differ from hazard ratios as these compare the mortality of specific dcSSc patients with the mortality of other dcSSc patients. The general population mortality data were extracted from the 2014
Italian life tables [18] as Italian was the most prevalent nationality $(22 \%)$ in the analyzed cohort from the EUSTAR database at the time of analysis [19]. This was used as a mortality multiplier, sensitive to the fact that a patient is in a specific HAQ-DI state or any advanced organ state.

After calibration, a microsimulation structure was constructed, which enabled patients to move between the five HAQ-DI states, to develop major advanced organ involvement, and/or to enter a death state. An illustrative microsimulation was proposed for a patient (simulated over a lifetime horizon) to randomly transition between the five HAQ-DI states, to develop lung/no lung involvement, and to enter a death state at each time cycle. The structure of the model is shown in Fig. 3. In that structure, a female patient starts in a specific HAQDI and lung state (defined by baseline characteristics). At the start of each cycle, based on her HAQ-DI state and lung state in the previous cycle, she could evolve towards another HAQ-DI and/or lung state, or die. The equations used to model the transitions in that illustrative model were:

\begin{tabular}{|c|c|c|c|c|c|c|}
\hline & $\begin{array}{l}\text { Number } \\
\text { at risk }\end{array}$ & $\begin{array}{c}\text { Observed } \\
\text { deaths }\end{array}$ & $\begin{array}{c}\text { Expected } \\
\text { deaths }\end{array}$ & SMR $[95 \% \mathrm{Cl}]$ & & \\
\hline \multicolumn{7}{|c|}{ Advanced Gl involvement } \\
\hline Yes & 596 & 135 & 22.34 & $6.04[5.67,6.44]$ & 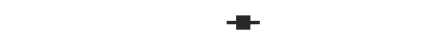 & \\
\hline No & 2716 & 537 & 82.35 & $6.52[6.33,6.72]$ & $=$ & \\
\hline \multicolumn{7}{|c|}{ Advanced renal involvement } \\
\hline Yes & 189 & 74 & 6.02 & $12.30[11.59,13.05]$ & & $\rightarrow-$ \\
\hline No & 3123 & 598 & 98.67 & $6.06[5.88,6.24]$ & $=$ & \\
\hline \multicolumn{7}{|c|}{ Advanced cardiac involvement } \\
\hline Yes & 171 & 69 & 8.17 & $8.45[7.88,9.05]$ & $\rightarrow$ & \\
\hline No & 3141 & 603 & 96.52 & $6.25[6.07,6.43]$ & $=$ & \\
\hline \multicolumn{7}{|c|}{ Advanced PH } \\
\hline Yes & 329 & 110 & 20.54 & $5.36[4.99,5.75]$ & $\rightarrow$ & \\
\hline No & 2983 & 562 & 84.15 & $6.68[6.48,6.88]$ & $=$ & \\
\hline \multicolumn{7}{|c|}{ Advanced lung involvement } \\
\hline Yes & 812 & 198 & 23.58 & $8.40[8.03,8.78]$ & 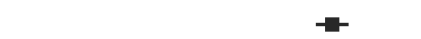 & \\
\hline No & 2500 & 474 & 81.11 & $5.84[5.65,6.04]$ & $=$ & \\
\hline \multicolumn{7}{|c|}{ HAQ-DI state } \\
\hline $2.0-3.0$ & 89 & 18 & 1.39 & $12.91[11.39,14.63]$ & & $\rightarrow$ \\
\hline $1.5-2.0$ & 87 & 6 & 0.97 & $6.17[4.49,8.48]$ & $\longrightarrow$ & \\
\hline $1.0-1.5$ & 86 & 3 & 1.18 & $2.54[1.28,5.02]$ & $\longrightarrow$ & \\
\hline $0.5-1.0$ & 76 & 1 & 0.87 & $1.14[0.19,6.85]$ & $\longrightarrow$ & \\
\hline $0.0-0.5$ & 128 & 5 & 1.43 & $3.50[2.22,5.54]$ & $\rightarrow$ & \\
\hline \multirow[t]{3}{*}{ Overall } & 3312 & 672 & 104.69 & $6.42[6.25,6.60]$ & $\nabla$ & \\
\hline & & & & & 10 & 15 \\
\hline & & & & & SMR & \\
\hline \multicolumn{7}{|c|}{$\begin{array}{l}\text { Fig. } 2 \text { Calculation of SMR using death rates from the EUSTAR dcSSC cohort (observed deaths) and deaths in the general population (expected } \\
\text { deaths from the Italian life tables) in patients with } \geq 1 \text { HAQ-DI score measurement. CI confidence interval, dCSSC diffuse cutaneous systemic } \\
\text { sclerosis, EUSTAR European Scleroderma Trials and Research, GI gastrointestinal, HAQ-DI Health Assessment Questionnaire-Disability Index, PH } \\
\text { pulmonary arterial hypertension, SMR standardized mortality ratio }\end{array}$} \\
\hline
\end{tabular}




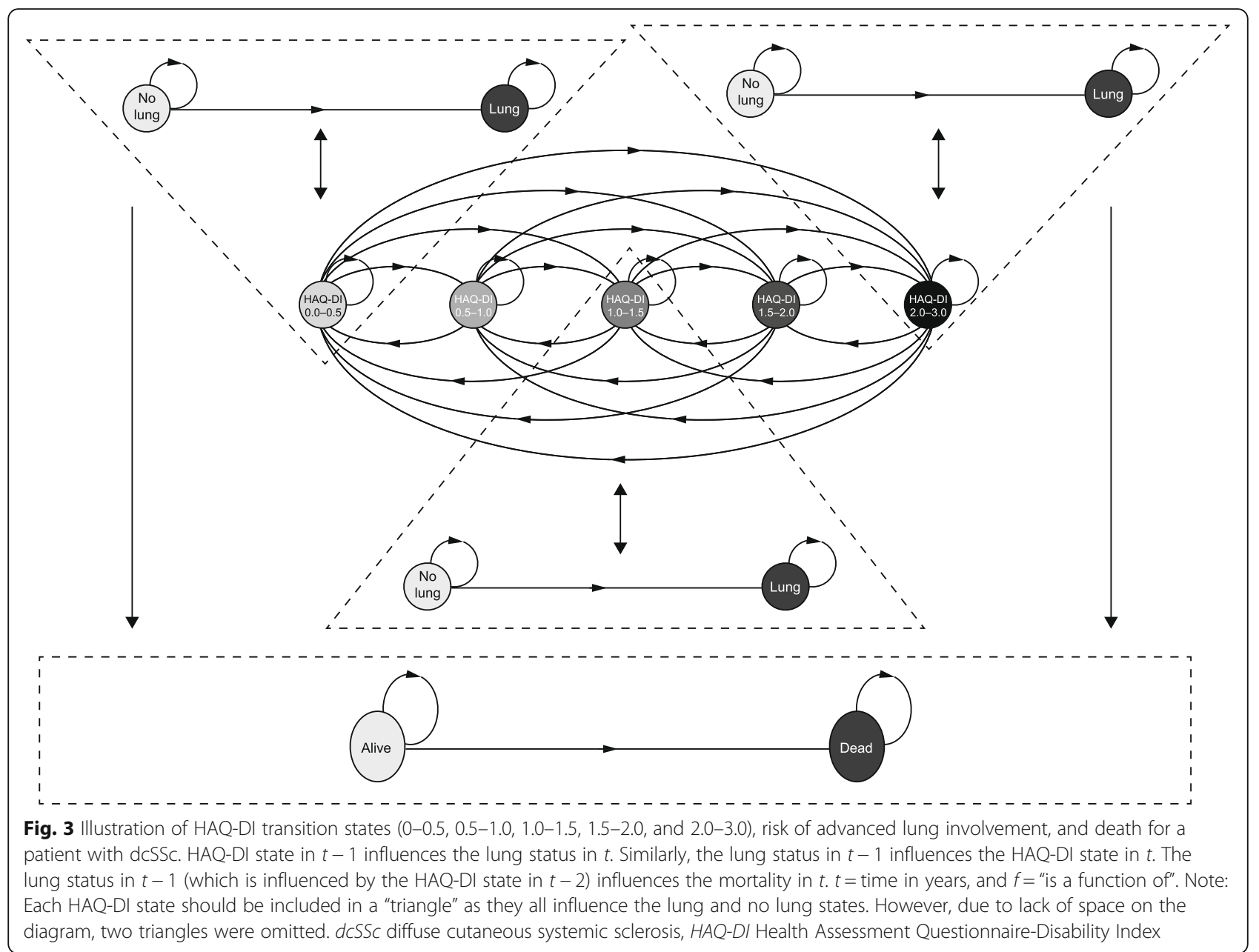

- HAQ-DI state in $t=f$ (age in $t-1$, sex, lung status in $t-1)$

- Lung status in $t=f(\mathrm{HAQ}-\mathrm{DI}$ state in $t-1$, time since disease onset)

- Mortality in $t=f$ (lung status in $t-1$, age in $t-1$, sex) where $t=$ time (years); $f=$ "is function of".

Several assumptions were made as these equations were calibrated on data with different time horizons (only lung status was a time-dependent predicted variable). The calibrated equations were:

- HAQ-DI state after 1 year $=f$ (age at baseline, sex, lung status at baseline)

- Lung status in year $t=f(\mathrm{HAQ}-\mathrm{DI}$ state at baseline, time since disease onset)

- Mortality in $t=f($ lung status in $t-1$, age in $t-1$, sex)

\section{Results}

\section{Study population}

A total of 4132 dcSSc patients without HAQ-DI score and $690 \mathrm{dcSSc}$ patients with HAQ-DI score assessments were included in the EUSTAR registry. Of the 690 patients, 424 patients $(61.4 \%)$ had $\geq 2$ HAQ-DI score assessments and were included in HAQ-DI score progression and death rate analyses, and 266 patients (38.6\%) had only one HAQ-DI score assessment and were included in the death rate analysis (Fig. 1). The proportion of patients with major advanced organ involvement is shown in Table 1 . One advanced organ involvement was reported in $14.2 \%$ of patients with no HAQ-DI score assessments, in $26.2 \%$ with $\geq 1$ HAQ-DI score assessment, and in $25.6 \%$ with $\geq 2$ HAQ-DI score assessments.

The baseline characteristics of patients with $\geq 1$ ( $N=$ $690)$ and $\geq 2(N=424)$ HAQ-DI score assessments are summarized in Table 1. In general, the demographic characteristics of these patients were comparable with those with no HAQ-DI score assessments $(N=4132)$. However, most patients with $\geq 1$ (77.5\%) and $\geq 2$ (77.1\%) HAQ-DI score assessments had received immunomodulator treatment, whereas only $32.2 \%$ of those with no HAQ-DI data had received treatment (Fig. S1). For patients with $\geq 2$ HAQ-DI scores, the mean (standard 
deviation [SD]) duration between first and second assessment of the HAQ-DI score was 11.8 (9.6) months.

\section{Predictors of death}

Of the 690 patients with $\geq 1$ HAQ-DI score assessment, $522(75.7 \%)$ had follow-up information with a median duration of 29.9 months (range 2.1-86.2 months), and 41 (7.9\%) patients died. Univariate analysis showed that baseline HAQ-DI score, older age at onset of Raynaud's phenomenon, corticosteroid treatment $>10 \mathrm{mg}$ /day, and advanced major organ involvement (other than renal involvement) were respectively associated with the risk of death (Table 2). Of the baseline HAQ-DI score, all eight domains were associated with mortality. When testing the eight domains in a multivariable model with stepwise backward selection, only the domains walking and dressing remained as predictors of mortality (Table S3).

Multivariable analysis showed that baseline HAQ-DI score, corticosteroid treatment $>10 \mathrm{mg} /$ day, and major advanced organ involvement (1 or multiple organs) remained predictive of death after adjustment on baseline parameters; a 1-point increase in baseline HAQ-DI score multiplied the risk of death by $2.7(p<0.001)$ and multiple advanced major organ involvement multiplied the risk of death by $2.8(p<0.05)$ (Table 3$)$.

\section{Predictors of HAQ-DI score progression}

Of patients who had $\geq 2$ HAQ-DI score assessments, HAQ-DI score progression after 1 year ( \pm 2 months) was monitored in $62.3 \% \quad(n=264 / 424)$; of those, $78.0 \%$

Table 2 Univariate Cox regression analysis of factors influencing risk of death in patients with $\geq 1 \mathrm{HAQ}-\mathrm{DI}$ measurement

\begin{tabular}{llll}
\hline Baseline parameter & HR & $\mathbf{9 5 \%}$ Cl for HR & $\boldsymbol{p}$ value \\
\hline Male gender & 1.15 & $0.57,2.35$ & 0.698 \\
HAQ-DI score & 3.01 & $1.98,4.60$ & $<0.001$ \\
Age at onset of RP & 1.03 & $1.00,1.05$ & 0.030 \\
mRSS & 1.02 & $0.99,1.05$ & 0.303 \\
RNA polymerase III positive & 0.04 & $0.00,11.11$ & 0.260 \\
Anti-topoisomerase I antibody & 0.92 & $0.45,1.88$ & 0.817 \\
Immunomodulator treatment & 0.86 & $0.45,1.63$ & 0.638 \\
Corticosteroids > 10 mg/day & 6.29 & $2.64,14.98$ & $<0.001$ \\
One advanced organ involvement & 3.93 & $1.99,7.78$ & $<0.001$ \\
Two advanced organ involvement & 8.43 & $3.40,20.91$ & $<0.001$ \\
Advanced lung involvement & 4.81 & $2.37,9.77$ & $<0.001$ \\
Advanced cardiac involvement & 6.24 & $1.47,26.50$ & 0.013 \\
Advanced Gl involvement & 2.22 & $1.06,4.64$ & 0.035 \\
Advanced PH involvement & 6.50 & $2.66,15.88$ & $<0.001$ \\
Advanced renal involvement & 1.12 & $0.15,8.13$ & 0.914 \\
\hline
\end{tabular}

Cl confidence interval, Gl gastrointestinal, $H A Q-D I$ Health Assessment Questionnaire-Disability Index, HR hazard ratio, mRSS Modified Rodnan Skin Score, $P H$ pulmonary arterial hypertension, $R P$ Raynaud's phenomenon
Table 3 Multivariable Cox regression analysis of factors affecting risk of death in patients with $\geq 1 \mathrm{HAQ}-\mathrm{DI}$ measurement

\begin{tabular}{llll}
\hline Baseline parameter & HR & $\mathbf{9 5 \%} \mathbf{C l}$ for HR & $\boldsymbol{p}$ value \\
\hline HAQ-DI score & 2.69 & $1.71,4.23$ & $<0.001$ \\
Age at onset of RP & 1.02 & $0.99,1.04$ & 0.177 \\
mRSS & 1.01 & $0.98,1.04$ & 0.527 \\
Male gender & 1.06 & $0.48,2.35$ & 0.888 \\
Immunomodulator treatment & 0.41 & $0.20,0.84$ & 0.014 \\
Corticosteroids > 10 mg/day & 5.41 & $2.10,13.97$ & $<0.001$
\end{tabular}

Advanced organ involvement (reference: no organ involvement)

\begin{tabular}{llll} 
One & 3.57 & $1.77,7.18$ & $<0.001$ \\
Multiple & 4.81 & $1.73,13.34$ & 0.003 \\
\hline
\end{tabular}

CI confidence interval, HAQ-DI Health Assessment Questionnaire-Disability Index, HR hazard ratio, mRSS Modified Rodnan Skin Score, RP Raynaud's phenomenon

(206/264) also had a HAQ-DI score assessment at 1 year. The mean (SD) change in HAQ-DI score from baseline to 1 year was $0.004(0.39)$ for all patients $(n=206), 0.02$ $(0.39)$ in those with no major advanced organ involvement after 1 year $(n=146),-0.06(0.38)$ in those with $\geq 1$ major advanced organ involvement after 1 year ( $n=24 / 55 ; 43.6 \%$ progressors), and $0.15(0.27)$ in those with $\geq 2$ major advanced organs involved $(n=3 / 5 ; 60.0 \%$ progressors).

On multivariable analysis (Table 4), patients with high baseline mRSS scores or esophageal symptoms showed

Table 4 Multivariable linear regression analysis of effect of baseline characteristics on HAQ-DI score progression at 1 year

\begin{tabular}{|c|c|c|c|}
\hline & $B$ & $95 \% \mathrm{Cl}$ for $B$ & $p$ value \\
\hline Constant & -0.16 & $-0.51,0.20$ & 0.389 \\
\hline HAQ-DI score at baseline & -0.15 & $-0.27,-0.04$ & 0.009 \\
\hline Age at onset of RP & -0.002 & $-0.01,0.004$ & 0.518 \\
\hline Male gender & 0.02 & $-0.18,0.22$ & 0.834 \\
\hline mRSS & 0.01 & $0.0001,0.03$ & 0.048 \\
\hline Immunomodulator treatment & 0.02 & $-0.17,0.20$ & 0.870 \\
\hline Corticosteroids > 10 mg/day & 0.12 & $-0.77,1.01$ & 0.791 \\
\hline Advanced organ involvement ${ }^{a}$ & -0.15 & $-0.67,0.37$ & 0.575 \\
\hline Advanced lung involvement & 0.05 & $-0.55,0.65$ & 0.866 \\
\hline Advanced cardiac involvement & 0.62 & $-0.36,1.60$ & 0.213 \\
\hline Advanced Gl involvement ${ }^{b}$ & 0.40 & $-0.20,0.99$ & 0.186 \\
\hline Advanced renal involvement & 0.47 & $-0.56,1.50$ & 0.367 \\
\hline Esophageal symptoms & 0.33 & $0.14,0.52$ & 0.001 \\
\hline Stomach symptoms & -0.04 & $-0.25,0.16$ & 0.670 \\
\hline Intestinal symptoms & 0.09 & $-0.09,0.26$ & 0.335 \\
\hline
\end{tabular}

Cl confidence interval, Gl gastrointestinal, HAQ-DI Health Assessment Questionnaire-Disability Index, mRSS modified Rodnan Skin Score, $R P$ Raynaud's phenomenon

${ }^{\mathrm{a}}$ Involved organs coded as $0=$ none, $1=$ one organ involvement, $2=$ multiple organ involvement

${ }^{\mathrm{b}}$ Malabsorption or $\geq 10 \%$ weight loss from baseline 
worsening function (i.e., increasing HAQ-DI scores) over 1 year, whereas those with higher baseline HAQ-DI scores showed improved functioning (i.e., decreasing HAQ-DI scores). Major advanced organ involvement at baseline was not predictive of HAQ-DI score progression at 1 year.

\section{Transition model for dcSSc}

The illustrative microsimulation model was run over 40 years (with a cycle length equal to 1 year) for a cohort of patients with dcSSc experiencing HAQ-DI states transitions, developing advanced lung involvement, and dying. The distribution of these patients over time between the various HAQ-DI, lung, and mortality states is shown in Fig. 4. This prediction was based on a simulated cohort of 1000 patients with the following baseline characteristics: $33 \%$ male; aged 50 years; HAQ-DI score $<0.5$; no lung involvement; and time elapsed since disease onset equal to 0 . dcSSc patients do not have a linear HAQ-DI score evolution; therefore, a transition-based model enabled a cohort of dcSSc patients to experience both improvement and worsening of HAQ-DI score. By modifying those baseline characteristics and simulating another dcSSc population (e.g., all patients starting in HAQ-DI score $>2.5$ ), one can observe how patients are differently distributed between the various health states along the simulation (e.g., they will experience advanced lung events faster and die sooner).

\section{Discussion}

The objectives of this EUSTAR database analysis were to identify predictors of death (with an emphasis on HAQDI score and major advanced organ involvement), to identify predictors of HAQ-DI score progression at 1 year, and to develop a model to predict disease progression in dcSSc using HAQ-DI score, major advanced organ involvement, and death rates. For death outcomes, the study showed that baseline HAQ-DI score, corticosteroid treatment, and advanced major organ involvement ( 1 or multiple organs) were predictive of death; importantly, the study showed that baseline HAQ-DI score and multiple major advanced organ involvement were equally and independently predictive of death. The link between corticosteroids and mortality risk is difficult to interpret, but they are commonly used for associated arthritis, myositis, or ILD, which all have poor prognosis. Therefore, a bias for indication may apply; corticosteroids $>10 \mathrm{mg} /$ day could be a surrogate indicator for more severe disease with a higher mortality risk.

For HAQ-DI score progression, patients with high baseline mRSS and esophageal symptoms showed worsening HAQ-DI score at 1 year, which may indicate that these measures correlate well in assessing disease progression in patients with dcSSc. Patients with a high baseline HAQ-DI score showed improved functioning, which could be a regression to the mean or may reflect a potential for improvement compared with those who had low HAQ-DI scores at baseline.

A previous study of patients with SSc included in the EUSTAR registry identified multiple predictors of low survival, including male sex, age $>50$ years, cardiac impairment (LVEF <50\%), and ILD, although HAQ-DI scores were not reported [20]. In the present study, we can clearly see the importance of the HAQDI score as a predictor of death in addition to these predictors (notably major advanced organ involvement); it is also a marker of disease progression that may correlate well with mRSS. This is also consistent with other studies where the HAQ was found to be predictive of mortality in the general population and in patients with rheumatoid arthritis and inflammatory polyarthritis [21-23].
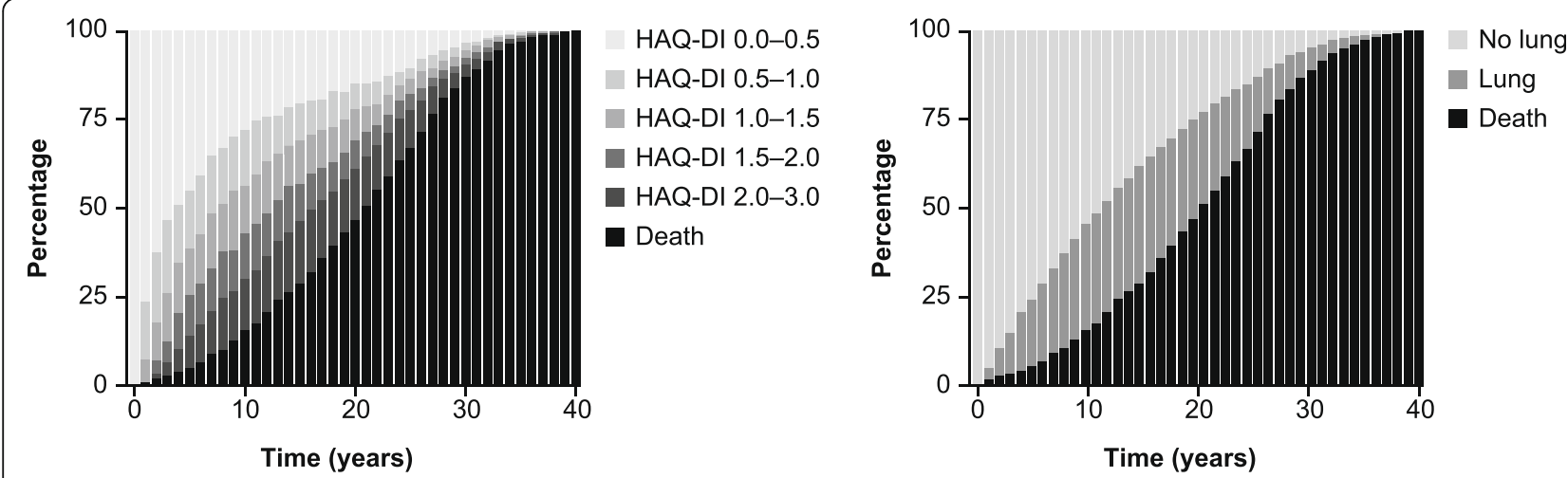

Fig. 4 Distribution of dcSSc patients over time between the various HAQ-DI, lung and mortality states as per an illustrative microsimulation that was run over 40 years for a cohort of dcSSc patients. Note: Model was based on 1000 simulated patients with specific baseline characteristics (33\% male, age 50 years, HAQ-DI score < 0.5, no lung involvement, and known time since disease onset). dcSSc diffuse cutaneous systemic sclerosis, HAQ-DI Health Assessment Questionnaire-Disability Index 
The clinical relevance of the findings from this study is multi-fold. First, this analysis may change physician perception about the HAQ-DI score, and they may now rate it on a comparable level to more objective measures, such as mRSS or organ involvement. Second, the HAQDI score could be used as an endpoint in clinical studies of patients with dcSSc. It has been used as an endpoint in recent studies with tocilizumab [8] and abatacept [9] in patients with SSc, and has been shown to be responsive to treatment-induced changes in disease activity. In one study, HAQ-DI score was used separately and as part of the ACR Combined Response Index in Systemic Sclerosis (ACR-CRISS, a composite endpoint that captures cardio-pulmonary-renal involvement and change in mRSS, HAQ-DI score, patient and physician global assessments, and FVC \% predicted) [9]; this has been used a primary outcome, and our findings support the value of HAQ-DI score. Third, the HAQ-DI score should be included in health technology assessments of new drug treatments in dcSSc. Many self-evaluations in clinical studies are now mandated by the US Food and Drug Administration [9]. The HAQ-DI score has also been supported in health technology appraisals by the National Institute for Health and Clinical Excellence for oral mycophenolate [24] and rituximab [25] in SSc. Finally, the transition model provides a useful way of monitoring treatment needs over a lifetime horizon, and it could be used in economic models to monitor the impact of new treatments in different patient subgroups.

The strengths of this study include the use of a large, prospective registry and rigorous documentation of $\mathrm{dcSSc}$ and advanced organ involvement. In this model, death rates were also calculated using an SMR. This is the first time that SMRs per organ involvement have been calculated, although it was not a key objective of the study. The SMRs were higher than reported in previous studies $[5,26]$. In one meta-analysis of 9 studies in 2691 patients with SSc, the pooled SMR was 3.53 (95\% CI 3.03, 4.11; $p<0.001)$. In another French study of 625 patients with SSc, overall SMR was 5.73 (95\% CI 4.68, 6.94). Taken together, these studies highlight the devastating nature of this condition.

Limitations include the observational design, lack of treatment effect on outcomes, and no inclusion of nonEuropean patients. Regarding the computation of SMRs, we observed a low number of deaths in each HAD-DI category, and this resulted in a non-linear progression of SMRs when increasing the HAQ-DI category. Therefore, this low number of deaths in each of the five HAQ-DI categories may introduce some instability in the results. In addition, the model shown was a simplified illustration of how this tool could work to monitor disease progression. It did not account for any other organ involvement, and only advanced lung involvement was considered a predictor of death (HAQ-DI score is a predictor of death, along with some other baseline characteristics, but these were not considered in order to avoid multicollinearity issues). As SMRs were calculated exclusively in dcSSc patients with non-missing HAQ-DI values or non-missing lung status, it is likely SMRs for dcSSc patients are slightly overestimated. Indeed, it is a known fact that patient in critical condition are more likely to have metabolic and questionnaire-related values recorded. A meta-analysis (nine studies from 1960 to 2010) among SSc patients (not exclusively dcSSc patients) presented an SMR equal to 3.53 (95\% CI 3.03, 4.11) [26]. This study also reported a non-significant change of SMR over the past 40 years. Therefore, we deemed the SMRs calculated among dcSSc patients as acceptable.

\section{Conclusions}

This EUSTAR database analysis showed that HAQ-DI scores offer a useful method of monitoring the natural progression of dcSSc in relation to major advanced organ involvement and death. The HAQ-DI score was found to be a significant predictor of mortality risk, thus highlighting the major importance of subjective measures on disease activity and patient well-being. It is hoped that this could evoke a paradigm switch to focusing on more global measures of disease activity in dcSSc, and to rate the HAQ-DI scores in comparison to more objective measures such as major advanced organ involvement. Overall, these findings support the importance of the HAQ-DI score as an endpoint in clinical studies of therapeutic interventions in dcSSc. This analysis also provides a useful model for predicting longterm disease progression in dcSSc.

\section{Supplementary information}

Supplementary information accompanies this paper at https://doi.org/10. 1186/s13075-020-02329-2.

Additional file 1: Fig. S1. Immunomodulator treatment received at baseline according to HAQ-DI score assessments. Table S1. Results of the multistate model calibration on longitudinal patient-level data (transition intensities between the HAQ-DI states in function of covariates).

Table S2. Results of a survival model calibration on longitudinal patientlevel data (transition intensities between the organ states in function of covariates). Table S3. Univariate and multivariable Cox regression analysis of the HAQ-DI domains influencing mortality risk in patients with $\geq 1$ HAQ-DI measurement.

\section{Abbreviations}

ACR: American College of Rheumatology; Cl: Confidence interval; dcSSc: Diffuse cutaneous systemic sclerosis; EULAR: European League Against Rheumatism; EUSTAR: European Scleroderma Trials and Research; Gl: Gastrointestinal; HAQ-DI: Health Assessment Questionnaire-Disability Index; HR: Hazard ratio; ILD: Interstitial lung disease; IQR: Interquartile range; LVEF: Left ventricular ejection fraction; mRSS: Modified Rodnan Skin Score; MSM: Multistate modelling; NA: Not available; PH: Pulmonary hypertension; 
RP: Raynaud's phenomenon; SD: Standard deviation; SE: Standard error; SMR: Standardized mortality ratio; SSc: Systemic sclerosis

\section{Acknowledgements}

Writing assistance was provided by Parexel International, UK, and was funded by Sanofi R\&D, France, in accordance with the Good Publication Practice 3 (GPP3) guidelines (http://www.ismpp.org/gpp3).

\section{Authors' contributions}

YA, SB, AT, DH, CA, and CS made substantial contributions to study concept and design. YA, ES, LC, PEC, EH, EZ, ML, PA, FAM, ER, and OD contributed to data acquisition. YA, SB, AT, and DH contributed to data analysis. All authors contributed to data interpretation, drafting the manuscript, and critical revision for important intellectual content. All authors approved the final version of the manuscript and are accountable for accuracy and integrity.

\section{Funding}

The study was sponsored by Sanofi R\&D, Chilly-Mazarin, France.

\section{Availability of data and materials}

The data that support the findings of this study are available from Professor Yannick Allanore, upon request. Further details on Sanofi's data sharing criteria, eligible studies, and process for requesting access can be found at: https://www.clinicalstudydatarequest.com.

\section{Ethics approval and consent to participate}

Ethics Committee approval and informed consent was provided when required by the ethical regulations of the specific centers.

\section{Consent for publication}

All the results presented in this article are anonymized and in aggregate form, and no personally identifiable information was used for this study.

\section{Competing interests}

Yannick Allanore received fees or grants from Actelion, Bayer, Boehringer Ingelheim, Bristol Myers Squibb, Curzion, Inventiva, Roche, and Sanofi. Oliver Distler had consultancy relationship and/or has received research funding from AbbVie, Actelion, Acceleron Pharma, Amgen, AnaMar, Baecon Discovery, Blade Therapeutics, Bayer, Boehringer Ingelheim, Catenion, Competitive Drug Development International Ltd., CSL Behring, ChemomAb, Curzion Pharmaceuticals, Ergonex, Galapagos NV, Glenmark Pharmaceuticals, GSK, Inventiva, Italfarmaco, iQone, iQvia, Lilly, Medac, Medscape, Mitsubishi Tanabe Pharma, MSD, Novartis, Pfizer, Roche, Sanofi, Target Bio Science, and UCB in the area of potential treatments of scleroderma and its complications. In addition, Oliver Distler has a patent mir-29 for the treatment of systemic sclerosis issued (US8247389, EP2331143).

Elise Siegert has received speaker fees/compensation for advisory board meetings from Actelion and Boehringer Ingelheim and a grant from Actelion

László Czirják receives honoraria for consulting services (educational services, scientific articles, participation in advisory boards, clinical trials, other) from AbbVie, Actelion, Boehringer Ingelheim, Lilly, MSD, Novartis, Pfizer, Richter, Roche, and UCB.

Patricia E Carreira received fees or grants from Actelion, Boehringer Ingelheim, Emmerald Health Pharmaceuticals, Sanofi, AbbVie, Bristol Myers Squibb, and Mitsubishi Tanabe Pharma.

Eric Hachulla has no conflicts of interest to declare.

Elisabetta Zanatta had consultancy relationship and/or received fees from Actelion and GSK.

Mengtao Li declares no conflict of interest.

Paolo Airò has received consultancy and/or travel expenses from

Bristol Myers Squibb, CSL Behring, Janssen, Novartis, Pfizer, Roche, and SOBI.

Fabian A Mendoza had a consultancy relationship with AbbVie, Genentech, and Gilead.

Edoardo Rosato has received research funding from Actelion.

Sylvie Bozzi, Augustin Terlinden, Caroline Amand, and Christina Soubrane are employees of Sanofi R\&D, France.

Doerte Huscher has received traveling expenses from Actelion Pharmaceuticals, Boehringer Ingelheim, and Shire International GmbH.

\section{Author details}

${ }^{1}$ Service de Rhumatologie, Cochin Hospital, APHP, Paris Descartes University, 27 rue du Faubourg Saint Jacques, 75014 Paris, France. ${ }^{2}$ Sanofi R\&D, Chilly-Mazarin, France. ${ }^{3}$ Institute of Biometry and Clinical Epidemiology and Berlin Institute of Health, Charité - Universitaetsmedizin, Berlin, Germany. ${ }^{4}$ Charité University Hospital, Berlin, Germany. ${ }^{5}$ University of Pécs, Medical School, Pécs, Hungary. ${ }^{6}$ Hospital University 12 October, Madrid, Spain. 7 University of Lille, Claude Huriez' Hospital, Lille Cedex, France. ${ }^{8}$ University of Padova, Padova, Italy. ${ }^{9}$ Peking Union Medical College Hospital, Chinese Academy of Medical Sciences, Beijing, P.R. China. ${ }^{10}$ Rheumatology and Clinical Immunology, Spedali Civili, Brescia, Italy. ${ }^{11}$ Jefferson Institute of Molecular Medicine, Thomas Jefferson University, Philadelphia, PA, USA. ${ }^{12}$ La Sapienza University, Polyclinic Umberto I, Rome, Italy. ${ }^{13}$ Department of Rheumatology, University Hospital Zurich, Zurich, Switzerland.

Received: 26 June 2020 Accepted: 25 September 2020

Published online: 28 October 2020

\section{References}

1. Denton CP, Khanna D. Systemic sclerosis. Lancet. 2017;390:1685-99.

2. Jaeger VK, Distler O, Maurer B, Czirjak L, Lorand V, Valentini G, et al. Functional disability and its predictors in systemic sclerosis: a study from the DeSScipher project within the EUSTAR group. Rheumatology (Oxford). 2018; 57:441-50.

3. Khanna D, Furst DE, Hays RD, Park GS, Wong WK, Seibold JR, et al. Minimally important difference in diffuse systemic sclerosis: results from the Dpenicillamine study. Ann Rheum Dis. 2006;65:1325-9.

4. LeRoy EC, Black C, Fleischmajer R, Jablonska S, Krieg T, Medsger TA Jr, et al. Scleroderma (systemic sclerosis): classification, subsets and pathogenesis. J Rheumatol. 1988;15:202-5.

5. Pokeerbux MR, Giovannelli J, Dauchet L, Mouthon L, Agard C, Lega JC, et al. Survival and prognosis factors in systemic sclerosis: data of a French multicenter cohort, systematic review, and meta-analysis of the literature. Arthritis Res Ther. 2019;21:86.

6. Khanna D, Furst DE, Clements PJ, Allanore Y, Baron M, Czirjak L, et al. Standardization of the modified Rodnan skin score for use in clinical trials of systemic sclerosis. J Scleroderma Relat Disord. 2017;2:11-8.

7. Wu W, Jordan S, Graf N, de Oliveira PJ, Curram J, Allanore Y, et al. Progressive skin fibrosis is associated with a decline in lung function and worse survival in patients with diffuse cutaneous systemic sclerosis in the European Scleroderma Trials and Research (EUSTAR) cohort. Ann Rheum Dis. 2019;78:648-56.

8. Khanna D, Denton CP, Jahreis A, van Laar JM, Frech TM, Anderson ME, et al. Safety and efficacy of subcutaneous tocilizumab in adults with systemic sclerosis (faSScinate): a phase 2, randomised, controlled trial. Lancet. 2016; 387:2630-40.

9. Khanna D, Spino C, Johnson S, Chung L, Whitfield M, Denton CP, et al. Abatacept in early diffuse cutaneous systemic sclerosis: results of a phase OO investigator-initiated, multicenter, double-blind randomized placebocontrolled trial. Arthritis Rheumatol. 2020;72:125-36.

10. Frantz C, Avouac J, Distler O, Amrouche F, Godard D, Kennedy AT, et al. Impaired quality of life in systemic sclerosis and patient perception of the disease: a large international survey. Seminar Arthritis Rheumatism. 2016;46: 115-23.

11. Fries JF, Spitz $P$, Kraines RG, Holman HR. Measurement of patient outcome in arthritis. Arthritis Rheum. 1980;23:137-45.

12. Pope J. Measures of systemic sclerosis (scleroderma): Health Assessment Questionnaire (HAQ) and Scleroderma HAQ (SHAQ), physician- and patientrated global assessments, Symptom Burden Index (SBI), University of California, Los Angeles, Scleroderma Clinical Trials Consortium Gastrointestinal Scale (UCLA SCTC GIT) 2.0, Baseline Dyspnea Index (BDI) and Transition Dyspnea Index (TDI) (Mahler's Index), Cambridge Pulmonary Hypertension Outcome Review (CAMPHOR), and Raynaud's Condition Score (RCS). Arthritis Care Res (Hoboken). 2011;63 Suppl 11:S98-111.

13. Johnson SR, Hawker GA, Davis AM. The health assessment questionnaire disability index and scleroderma health assessment questionnaire in scleroderma trials: an evaluation of their measurement properties. Arthritis Rheum. 2005;53:256-62.

14. Müller-Ladner U, Tyndall A, Czirjak L, Denton C, Matucci-Cerinic M, Centres E. Ten years EULAR scleroderma research and trials (EUSTAR): what has been achieved? Ann Rheum Dis. 2014;73:324-7. 
15. Wirz EG, Jaeger VK, Allanore Y, Riemekasten G, Hachulla E, Distler O, et al. Incidence and predictors of cutaneous manifestations during the early course of systemic sclerosis: a 10-year longitudinal study from the EUSTAR database. Ann Rheum Dis. 2016;75:1285-92.

16. van den Hoogen F, Khanna D, Fransen J, Johnson SR, Baron M, Tyndall A, et al. 2013 classification criteria for systemic sclerosis: an American college of rheumatology/European league against rheumatism collaborative initiative. Ann Rheum Dis. 2013;72:1747-55.

17. Preliminary criteria for the classification of systemic sclerosis (scleroderma). Subcommittee for scleroderma criteria of the American Rheumatism Association Diagnostic and Therapeutic Criteria Committee. Arthritis Rheum. 1980;23:581-90.

18. Statistisches Bundesamt. Deaths, life expectancy. https://www.destatis.de/ EN/Themes/Society-Environment/Population/Deaths-Life-Expectancy/_node. $\mathrm{html}$. Accessed 1 June 2020.

19. Human Mortality Database. The Human Mortality Database. https://www. mortality.org/. Accessed 1 June 2020.

20. Elhai M, Meune C, Boubaya M, Avouac J, Hachulla E, Balbir-Gurman A, et al. Mapping and predicting mortality from systemic sclerosis. Ann Rheum Dis. 2017;76:1897-905.

21. Farragher TM, Lunt M, Bunn DK, Silman AJ, Symmons DP. Early functional disability predicts both all-cause and cardiovascular mortality in people with inflammatory polyarthritis: results from the Norfolk Arthritis Register. Ann Rheum Dis. 2007;66:486-92.

22. Sokka T, Häkkinen A, Krishnan E, Hannonen P. Similar prediction of mortality by the health assessment questionnaire in patients with rheumatoid arthritis and the general population. Ann Rheum Dis. 2004;63:494-7.

23. Wolfe F, Michaud K, Gefeller O, Choi HK. Predicting mortality in patients with rheumatoid arthritis. Arthritis Rheum. 2003;48:1530-42.

24. National Institute for Health and Care Excellence. Scleroderma: oral mycophenolate. Published 29 July 2014. Available at: https:/www.nice.org. uk/advice/esuom32/resources/scleroderma-oral-mycophenolatepdf-54116459058683077. Accessed 1 June 2020.

25. National Institute for Health and Care Excellence. Skin involvement in systemic sclerosis: rituximab. Published 7 March 2017. Available at: https:// www.nice.org.uk/advice/es7/resources/skin-involvement-in-systemicsclerosis-rituximab-32179717. Accessed 1 June 2020.

26. Elhai M, Meune C, Avouac J, Kahan A, Allanore Y. Trends in mortality in patients with systemic sclerosis over 40 years: a systematic review and meta-analysis of cohort studies. Rheumatology (Oxford). 2012;51:1017-26.

\section{Publisher's Note}

Springer Nature remains neutral with regard to jurisdictional claims in published maps and institutional affiliations.

Ready to submit your research? Choose BMC and benefit from:

- fast, convenient online submission

- thorough peer review by experienced researchers in your field

- rapid publication on acceptance

- support for research data, including large and complex data types

- gold Open Access which fosters wider collaboration and increased citations

- maximum visibility for your research: over $100 \mathrm{M}$ website views per year

At BMC, research is always in progress.

Learn more biomedcentral.com/submissions 